ANNALES

POLONICI MATHEMATICI

$85.1(2005)$

\title{
Estimates of weak solutions to nondiagonal quasilinear parabolic systems
}

\author{
by Dmitry Portnyagin (Lviv)
}

\begin{abstract}
L^{\infty}$-estimates of weak solutions are established for a quasilinear nondiagonal parabolic system with a special structure whose leading terms are modelled by $p$-Laplacians. A generalization of the weak maximum principle to systems of equations is employed.
\end{abstract}

1. Introduction. In the present paper we study the boundedness of weak solutions to a quasilinear nondiagonal parabolic system of equations in divergence form under special assumptions imposed upon its structure.

It is well known that the De Giorgi-Nash-Moser estimates are no longer valid for a general elliptic system, for any kind of ellipticity. Such a system can be regarded as a special case of a parabolic system, when partial temporal derivatives of unknowns are identically zero. An example of an unbounded solution to a linear elliptic system with bounded coefficients was given by De Giorgi in [8]:

$$
\sum_{i=1}^{n} \frac{\partial}{\partial x_{i}}\left(\sum_{j=1}^{n} \sum_{\alpha=1}^{n} A_{i j}^{\alpha \beta}(x) \frac{\partial}{\partial x_{j}} u_{\beta}\right)=0, \quad \beta=1, \ldots, n, n \geq 3,
$$

under the following hypotheses upon the matrix of coefficients:

$$
\begin{gathered}
A_{i j}^{\alpha \beta}(x)=\delta_{\alpha \beta} \delta_{i j}+\left[(n-2) \delta_{\alpha i}+n \frac{x_{i} x_{\alpha}}{|x|^{2}}\right] \cdot\left[(n-2) \delta_{\beta j}+n \frac{x_{j} x_{\beta}}{|x|^{2}}\right] \\
\delta_{\alpha \beta}= \begin{cases}1, & \alpha=\beta, \\
0, & \alpha \neq \beta ;\end{cases}
\end{gathered}
$$

2000 Mathematics Subject Classification: Primary 35B50; Secondary 35K40, 35K55.

Key words and phrases: nondiagonal parabolic systems, bounded solution, Dirichlet data.

This paper was written during the author's being a Ph.D. student at the Institute of Mathematics of the Polish Academy of Sciences, whose financial support is gratefully acknowledged. 


$$
\sum_{i=1}^{n} \sum_{j=1}^{n} \sum_{\alpha=1}^{n} \sum_{\beta=1}^{n} A_{i j}^{\alpha \beta}(x) \xi_{\alpha}^{i} \xi_{\beta}^{j} \geq|\xi|^{2} \quad \text { for all } \xi \in \mathbb{R}^{2 n}, x \in \mathbb{R}^{n}
$$

it is easy to check that the vector-valued function $u(x)=x /|x|^{\gamma}$ with $\gamma=$ $(n / 2)\left(1-\left[(2 n-2)^{2}+1\right]^{-1 / 2}\right)$ is an unbounded weak solution to the system in the domain $\Omega=\{x|| x \mid \leq 1\} \subset \mathbb{R}^{n}$, though its coefficients remain bounded, $A_{i j}^{\alpha \beta} \in L^{\infty}(\Omega)$.

There is yet another example due to J. Nečas and J. Souček of a nonlinear elliptic system with sufficiently smooth coefficients, but with a weak solution not belonging to $W^{2,2}$ :

$$
\sum_{i=1}^{n} \frac{\partial}{\partial x_{i}}\left(\sum_{j=1}^{n} \sum_{\alpha=1}^{n} A_{i j}^{\alpha \beta}(x, u) \frac{\partial}{\partial x_{j}} u_{\beta}\right)=0, \quad \beta=1, \ldots, n, n \geq 5,
$$

where

$$
\begin{gathered}
A_{i j}^{\alpha \beta}(x, u)=\delta_{\alpha \beta} \delta_{i j}+c^{2}\left[\delta_{\alpha i}+b \frac{u^{i} u^{\alpha}|x|^{2 \gamma-2}}{1+|u|^{2}|x|^{2 \gamma-2}}\right] \cdot\left[\delta_{\beta j}+b \frac{u^{j} u^{\beta}|x|^{2 \gamma-2}}{1+|u|^{2}|x|^{2 \gamma-2}}\right], \\
b=2 n /(n-2), \quad c^{2}=\gamma(n-\gamma)(n-2)^{2} /\left[(n-2 \gamma)^{2}(n-1)^{2}\right]
\end{gathered}
$$

and, obviously, for all $\xi \in \mathbb{R}^{2 n}, x \in \mathbb{R}^{n}, u \in \mathbb{R}^{n}$,

$$
\sum_{i=1}^{n} \sum_{j=1}^{n} \sum_{\alpha=1}^{n} \sum_{\beta=1}^{n} A_{i j}^{\alpha \beta}(x, u) \xi_{\alpha}^{i} \xi_{\beta}^{j} \geq|\xi|^{2}
$$

the vector-valued function $u(x)=x /|x|^{\gamma} \in W^{1,2}(\Omega)$ with $\gamma \in[(n-2) / 2, n / 2)$ is a weak solution in $\Omega=\{x|| x \mid \leq 1\} \subset \mathbb{R}^{n}$ which, however, does not even belong to $W^{2,2}(\Omega)$, although the coefficients $A_{i j}^{\alpha \beta}(x, u)$ are sufficiently smooth.

These two examples, as well as many others, illustrate the fact that the regularity problem for elliptic systems proves to be far more complicated than for second order elliptic equations.

Until now a priori estimates of De Giorgi type have been extended only to a special class of parabolic systems, the so-called weakly coupled systems. A system is said to be weakly coupled if it is coupled only through the terms which are not differentiated, each equation containing derivatives of just one component.

There exists yet another approach to a priori estimates for a parabolic system of second order differential equations [14]. It concerns not each component separately, but the sum of the squares of the components of a solution. This applies to diagonal systems which on freezing the leading coefficients and discarding the right-hand sides and lower order terms reduce to just one single equation rewritten several times for all the unknown functions; see also [6, p. 27], [5, pp. 32-33], [4]. 
An extensive study of the existence and regularity properties of solutions to nonlinear strongly coupled systems of equations with coefficients depending on spatial coordinates and unknowns was given by H. Amann (see [1], [3], [2] and the vast bibliography therein). The technique used there relates to semigroups and is not extendable to equations with coefficients depending upon derivatives.

On the other hand, in the theory of scalar second order PDE's the most known and powerful tool for obtaining estimates is a special property of solutions, called the maximum principle.

Our results here are based upon a certain property of the solution to a system of two equations, which immediately yields bounds for the components of the solution. Since this property enables us to obtain a priori estimates of the solution of a system, just as the maximum principle does for the case of a single equation, we propose to call it the maximum principle for a system. The extension of the maximum principle property to a system of two equations has been expounded in our previous paper [15].

The main idea we are utilizing has been employed earlier in [16] for semilinear systems (see also [10], [17] and [13]), and consists in switching to two new functions of unknowns. For each such function an $L^{\infty}$ estimate is established in the conventional way, and hence the final conclusion about each component of the vector function solution is inferred. It turns out that this technique allows for extension to nondiagonal systems with nonlinearities in the spatial derivatives also.

In the present paper we further employ this method. Namely, restricting ourselves to systems of two second order equations in divergence form possessing a special structure, we obtain $\|u\|_{L^{\infty}(Q)}$ estimates for quasilinear degenerate parabolic systems of equations in divergence form, in which coupling occurs in the leading derivatives and whose leading coefficients depend on spatial coordinates $x$, unknowns $u$, and their spatial derivatives $u_{x}$.

2. Basic notations and hypotheses. We shall be considering a system of two equations of the form

$$
\left\{\begin{array}{l}
u_{t}-\frac{\partial}{\partial x_{i}}\left(A_{i}^{(1)}\left(x, u, v, u_{x}, v_{x}\right)\right)=B^{(1)}\left(x, u, v, u_{x}, v_{x}\right), \\
v_{t}-\frac{\partial}{\partial x_{i}}\left(A_{i}^{(2)}\left(x, u, v, u_{x}, v_{x}\right)\right)=B^{(2)}\left(x, u, v, u_{x}, v_{x}\right), \quad x \in Q,
\end{array}\right.
$$

in a cylindrical domain $Q$, with the Dirichlet boundary conditions, which are understood in the weak sense:

$$
\left\{\begin{array}{l}
\left(u-g_{1}, v-g_{2}\right)(x, t) \in W_{0}^{1, p}(\Omega) \quad \text { a.e. } t \in(0, T), \\
(u, v)(x, 0)=(u, v)_{0}(x) .
\end{array}\right.
$$


A model is a system with the following structure:

$$
\left\{\begin{array}{c}
u_{t}-\frac{\partial}{\partial x_{i}}\left(a_{1}(u, v)|\nabla u|^{p-2} u_{x_{i}}\right)-\frac{\partial}{\partial x_{i}}\left(b_{1}(u, v)|\nabla v|^{p-2} v_{x_{i}}\right)=f_{1}, \\
v_{t}-\frac{\partial}{\partial x_{i}}\left(a_{2}(u, v)|\nabla u|^{p-2} u_{x_{i}}\right) \\
-\frac{\partial}{\partial x_{i}}\left(b_{2}(u, v)|\nabla v|^{p-2} v_{x_{i}}\right)=f_{2}, \quad x \in Q .
\end{array}\right.
$$

A solution to system (2.1) with Dirichlet data (2.2) is understood in the weak sense, as in [9].

Definition 2.1. A measurable vector function $\left(u^{1}, u^{2}\right)=(u, v)$ is called a weak solution of problem (2.1)-(2.2) if

$$
u^{j} \in C\left(0, T ; L^{2}(\Omega)\right) \cap L^{p}\left(0, T ; W^{1, p}(\Omega)\right)
$$

and for all $t \in(0, T]$,

$$
\begin{aligned}
\int_{\Omega} u^{j} \varphi_{j}(x, t) d x+\iint_{\Omega \times(0, t]}\left\{-u^{j} \varphi_{j t}+A_{i}^{(j)} \varphi_{j x_{i}}\right\} d x d \tau & \\
& =\int_{\Omega} u_{0}^{j} \varphi_{j}(x, 0) d x+\iint_{\Omega \times(0, t]} B^{(j)} \varphi_{j} d x d \tau
\end{aligned}
$$

for all test functions

$$
\varphi \in C\left(0, T ; L^{2}(\Omega)\right) \cap L^{p}\left(0, T ; W_{0}^{1, p}(\Omega)\right), \quad \varphi \geq 0 .
$$

The boundary condition in (2.2) is meant in the weak sense.

This definition can also be reformulated in terms of the Steklov averages (see e.g. [9]). We shall use the notations:

Definition 2.2. Let $u \in C\left(0, T ; L^{2}(\Omega)\right) \cap L^{p}\left(0, T ; W^{1, p}(\Omega)\right)$ and $\varphi_{+}=$ $\max [\varphi, 0]$. We define

$$
\begin{aligned}
& \sup u=\inf \left\{l \mid(u-l)_{+} \in W_{0}^{1, p}(\Omega) \text { for a.e. } t \in(0, T)\right. \text { and } \\
& \left.(u-l)_{+}=0 \text { a.e. in }\{0\} \times \Omega\right\} ; \\
& \sup u=\inf \left\{l \mid(u-l)_{+}=0 \text { a.e. in } Q\right\} \text {; } \\
& Q \\
& \inf _{\partial Q} u=\sup \left\{l \mid(l-u)_{+} \in W_{0}^{1, p}(\Omega) \text { for a.e. } t \in(0, T)\right. \text { and } \\
& \left.(l-u)_{+}=0 \text { a.e. in }\{0\} \times \Omega\right\} ; \\
& \inf _{Q} u=\sup \left\{l \mid(l-u)_{+}=0 \text { a.e. in } Q\right\} .
\end{aligned}
$$

Let us also define the boundary norms of functions that will come in useful in the following considerations. 
DEFINITION 2.3. Let $\Omega$ be a domain in $\mathbb{R}^{n}$ and $\partial \Omega$ a portion of its boundary; let $H(\Omega)$ be a Sobolev space. For a function $u$ defined on $\partial \Omega$ we set

$$
\|u\|_{H(\partial \Omega)}=\inf _{\psi}\|\psi\|_{H(\Omega)},
$$

where the infimum is taken over all functions $\psi \in H(\Omega)$ such that $\psi(x)=$ $u(x)$ a.e. on $\partial \Omega$. We shall denote by $H(\partial \Omega)$ the function space for which the aforementioned norm is finite.

Let us describe the quantities and functions entering systems (2.1)-(2.3) as well as those that will appear later on.

The coefficients $a_{1,2}$ and $b_{1,2}$ in the model system (2.3) are bounded measurable $\mathbb{R} \times \mathbb{R} \rightarrow \mathbb{R}$ functions of $u$ and $v$ to be determined more precisely below; $Q=(0, T] \times \Omega ; S=\partial \Omega \times(0, T] ; \Omega$ is a bounded domain in $\mathbb{R}^{n} ; x \in \Omega$; $T>0 ; t \in(0, T] ; n>p \geq 2 ; i=1, \ldots, n$ and the summation convention over repeated indices is assumed; $u, v \in C\left(0, T ; L^{2}(\Omega)\right) \cap L^{p}\left(0, T ; W^{1, p}(\Omega)\right)$; $f_{1,2}=f_{1,2}(x) ; W_{0}^{1, p}(\Omega)$ is the space of functions in $W^{1, p}(\Omega)$ vanishing on $\partial \Omega$ in the sense of traces for a.e. $t \in(0, T]$. Throughout the paper $\nabla$ stands for differentiation with respect to spatial variables.

By parabolicity of system (2.1) it is meant that the part without derivatives with respect to time is elliptic. The notion of ellipticity for a system of second order differential equations is understood in the following sense, as introduced in [7]: there are $\lambda>0$ and $0<F \in L^{p /(p-1)}(Q)$ such that for all $s_{j}^{i} \in \mathbb{R}^{2 n}, r_{j} \in \mathbb{R}^{2}, x \in \mathbb{R}^{n}, j=1,2, i=1, \ldots, n$,

$$
A_{i}^{(j)}(x, r, s) s_{j}^{i} \geq \lambda|s|^{p}-F(j) .
$$

It should be emphasized that neither the Legendre nor the LegendreHadamard condition is imposed. The Legendre condition stems from the calculus of variations, the problem of minimization of a functional, as a sufficient condition for the existence of an extremal. Since it is to be calculated on the extremal it bears no relation to the set-up of the problem. Its usage as an ellipticity condition in the theory of systems of differential equations is entirely technically motivated, and it is applied mostly in the so-called indirect methods and in the study of partial regularity to meet the requirements of Caccioppoli's inequality (see e.g. [11]). The Legendre-Hadamard condition, which is a weakened version of the Legendre condition, has been regarded by many authors as a more natural ellipticity condition for systems. When the coefficients are continuous the Legendre-Hadamard condition suffices to derive the full regularity, but it is not enough for systems with bounded measurable coefficients or coefficients depending on the unknowns, because the technique used relies heavily upon Caccioppoli's inequality [12]. Our reason to dispense with either condition is that they both produce an obstacle from the technical point of view in the approach we take, and that is why 
we adopt the ellipticity condition (2.4) for quasilinear systems as the most appropriate for our ends.

In what follows for brevity we shall use the notations:

$$
\begin{aligned}
& \widetilde{u}_{0}= \begin{cases}u_{0}(x), & x \in \Omega, t=0, \\
g_{1}(x, t), & x \in \partial \Omega, t \in(0, T) ;\end{cases} \\
& \widetilde{v}_{0}= \begin{cases}v_{0}(x), & x \in \Omega, t=0, \\
g_{2}(x, t), & x \in \partial \Omega, t \in(0, T) .\end{cases}
\end{aligned}
$$

Let us introduce in addition the following function space:

DEFINITION 2.4.

$$
\widetilde{W}(Q)=L^{p^{\prime}}\left(W^{1, p^{\prime}}(0, T) ; \Omega\right) \cap L^{p}\left(0, T ; W^{1, p}(\Omega)\right), \quad p^{\prime}=\frac{p}{p-1} ;
$$

i.e. the function $u$ belongs to $\widetilde{W}(Q)$ if

$$
\int_{0}^{T} \int_{\Omega}\left(\left|u_{t}\right|^{p^{\prime}}+|\nabla u|^{p}+|u|^{p}+|u|^{p^{\prime}}\right)<\infty .
$$

We shall assume that the functions $g_{1,2}(x, t)$ and $(u, v)_{0}(x)$ in the boundary data (2.2) are such that

$$
\widetilde{u}_{0} \in \widetilde{W}(\partial Q), \quad \widetilde{v}_{0} \in \widetilde{W}(\partial Q),
$$

and in addition

$$
g_{1,2}(x, t) \in L^{\infty}(S), \quad(u, v)_{0}(x) \in L^{\infty}(\Omega) .
$$

About the leading parts $A_{i}^{(1),(2)}(x, r, s)$ it is assumed that they are measurable $\Omega \times \mathbb{R}^{2} \times \mathbb{R}^{2 n} \rightarrow \mathbb{R}$ functions that satisfy the ellipticity condition (2.4) and are subject to the following growth conditions: there exists $\lambda>0$ such that for all $s_{i}^{j} \in \mathbb{R}^{2 n}, r^{j} \in \mathbb{R}^{2}, x \in \mathbb{R}^{n}, j=1,2, i=1, \ldots, n$,

$$
\left|A_{i}^{(1),(2)}(x, r, s)\right| \leq \lambda|s|^{p-1} .
$$

For the model system the leading coefficients $A_{i}^{(1)}$ and $A_{i}^{(2)}$ are

$$
\begin{aligned}
& A_{i}^{(1)}(x, r, s)=a_{1}(r)\left|\vec{s}^{(1)}\right|^{p-2} s_{i}^{(1)}+b_{1}(r)\left|\vec{s}^{(2)}\right|^{p-2} s_{i}^{(2)}, \\
& A_{i}^{(2)}(x, r, s)=a_{2}(r)\left|\vec{s}^{(1)}\right|^{p-2} s_{i}^{(1)}+b_{2}(r)\left|\vec{s}^{(2)}\right|^{p-2} s_{i}^{(2)} .
\end{aligned}
$$

Let us now outline more precisely the conditions satisfied by $a_{1,2}$ and $b_{1,2}$. For one thing, there exist real numbers $A_{1}-A_{4}$ and $B_{1}-B_{4}$ such that for all $(u, v) \in \mathbb{R} \times \mathbb{R}$,

$$
\begin{array}{ll}
A_{1} \leq a_{1}(u, v) \leq A_{2}, & A_{3} \leq a_{2}(u, v) \leq A_{4} \\
B_{1} \leq b_{1}(u, v) \leq B_{2}, & B_{3} \leq b_{2}(u, v) \leq B_{4} .
\end{array}
$$


and in addition there exists $\lambda_{0}>0$ such that

$$
\begin{aligned}
\min \left\{\operatorname { i n f } _ { r } \left[a_{1}(r)-\right.\right. & \left.(1 / p) b_{1}(r)-(1-(1 / p)) a_{2}(r)\right], \\
& \left.\inf _{r}\left[b_{2}(r)-(1 / p) a_{2}(r)-(1-(1 / p)) b_{1}(r)\right]\right\} \geq \lambda_{0} .
\end{aligned}
$$

Next, the coefficients $a_{1,2}(u, v), b_{1,2}(u, v)$ in the model system (2.3) and the structure hypotheses $(2.7 \mathrm{a})-(2.7 \mathrm{~b})$ below are such that the following conditions are satisfied:

$$
\begin{aligned}
& A=\left(\frac{a_{1}}{b_{1}}\right)_{u}^{\prime}=\left(\frac{a_{1}}{b_{1}}\right)_{v}^{\prime}, \\
& (2.6 \mathrm{~b}) \quad B=\left(\frac{b_{2}}{b_{1}}\right)^{2}\left(\frac{a_{1}}{b_{2}}\right)_{u}^{\prime}+\left(\frac{a_{2}}{b_{1}}\right)_{u}^{\prime}=\left(\frac{b_{2}}{b_{1}}\right)^{2}\left(\frac{a_{1}}{b_{2}}\right)_{v}^{\prime}+\left(\frac{a_{2}}{b_{1}}\right)_{v}^{\prime}, \\
& \quad C=\left(\frac{b_{2}}{b_{1}}\right)^{2}\left(\frac{a_{2}}{b_{2}}\right)_{u}^{\prime}=\left(\frac{b_{2}}{b_{1}}\right)^{2}\left(\frac{a_{2}}{b_{2}}\right)_{v}^{\prime} \\
& \text { (2.6c) } \quad D(r)=\sqrt{B^{2}-4 A C}>0, \quad k_{1,2}=\frac{-B \pm \sqrt{B^{2}-4 A C}}{2 A}, \\
& \text { (2.6d) } \quad \forall r^{j} \in \mathbb{R}^{2}: \quad k_{1,2}^{p}+\frac{b_{2}(r)}{b_{1}(r)} k_{1,2}^{p-1}-\frac{a_{1}(r)}{b_{1}(r)} k_{1,2}-\frac{a_{2}(r)}{b_{1}(r)}=0, \\
& \quad \Lambda_{1,2}(r)=b_{1}(r) k_{1,2}+b_{2}(r)>0 .
\end{aligned}
$$

Finally, upon the leading parts of the system the following structure conditions (2.7a) $-(2.7 \mathrm{~b})$ are imposed.

There exist measurable functions $a_{1,2}(r), b_{1,2}(r)$, satisfying assumptions (2.6a)-(2.6d), and $F_{1,2}(x)$, along with real numbers $\varepsilon_{1,2}$ such that

$$
\left|\varepsilon_{1,2}\right| \leq \frac{\delta^{*} \min \left(\lambda_{0}, \Lambda_{1}, \Lambda_{2}\right)}{8 \max \left(1,\left|k_{1}\right|+\left|k_{2}\right|\right)}
$$

and for all $s_{i}^{(j)} \in \mathbb{R}^{2 n}, r^{j} \in \mathbb{R}^{2}, x \in \mathbb{R}^{n}, j=1,2, i=1, \ldots, n$ :

$$
\begin{aligned}
&\left|A_{i}^{(1)}(x, r, s)-\left(a_{1}(r)\left|s^{(1)}\right|^{p-2} s_{i}^{(1)}+\left.b_{1}(r)\left|s^{(2)}\right|\right|^{p-2} s_{i}^{(2)}\right)\right| \\
& \leq \theta\left(-\varepsilon_{1}\left|k_{2}\right|\left|k_{1} s^{(1)}+s^{(2)}\right|-\varepsilon_{2}\left|k_{1}\right|\left|k_{2} s^{(1)}+s^{(2)}\right|\right)\left(\left|s^{(1)}\right|^{p-2}+\left|s^{(2)}\right|^{p-2}\right) \\
& \quad \max \left[\varepsilon_{1}\left|k_{1} s^{(1)}+s^{(2)}\right|+\varepsilon_{2}\left|k_{2} s^{(1)}+s^{(2)}\right|, 0\right]+F_{1}(x), \\
&\left|A_{i}^{(2)}(x, r, s)-\left(a_{2}(r)\left|s^{(1)}\right|^{p-2} s_{i}^{(1)}+b_{2}(r)\left|s^{(2)}\right|^{p-2} s_{i}^{(2)}\right)\right| \\
& \leq \theta\left(\varepsilon_{1}\left|k_{1} s^{(1)}+s^{(2)}\right|+\varepsilon_{2}\left|k_{2} s^{(1)}+s^{(2)}\right|\right)\left(\left|s^{(1)}\right|^{p-2}+\left|s^{(2)}\right|^{p-2}\right) \\
& \quad \times \max \left[-\varepsilon_{1}\left|k_{2}\right|\left|k_{1} s^{(1)}+s^{(2)}\right|-\varepsilon_{2}\left|k_{1}\right|\left|k_{2} s^{(1)}+s^{(2)}\right|, 0\right]+F_{2}(x),
\end{aligned}
$$

where we have set $\Lambda_{1}=\inf _{r} \Lambda_{1}(r), \Lambda_{2}=\inf _{r} \Lambda_{2}(r) ;\left(k_{1}, \Lambda_{1}(r)\right)$ and $\left(k_{2}, \Lambda_{2}(r)\right)$ are from $(2.6 \mathrm{~d})$ or, equivalently, are two distinct solutions to 
the system

$$
\left\{\begin{array}{l}
k a_{1}(r)+b_{1}(r)=\Lambda(r)|k|^{p-2} k \\
k a_{2}(r)+b_{2}(r)=\Lambda(r), \quad \Lambda(r)>0
\end{array}\right.
$$

and $\lambda_{0}$ is from (2.6a).

REMARK 1 . It is easy to check that under assumption (2.6a), the ellipticity condition (2.4) holds for the model system with $\lambda=\lambda_{0}$. One may also see that the structure conditions $(2.7 \mathrm{a})-(2.7 \mathrm{~b})$ imply the ellipticity condition (2.4) with $\lambda=\lambda_{0}\left(1-\delta^{*} / 2\right)$ and $F \equiv|\eta|\left(\left|F_{1}\right|+\left|F_{2}\right|\right), \eta$ a number.

About the right-hand sides $B^{(1),(2)}(x, r, s)$ it is assumed that they are measurable $\Omega \times \mathbb{R} \times \mathbb{R} \times \mathbb{R}^{n} \times \mathbb{R}^{n} \rightarrow \mathbb{R}$ functions such that there exist $0<\varepsilon<p^{2} /(n+p)$ and $\Lambda_{2}>0$ such that for all $s_{i}^{(j)} \in \mathbb{R}^{2 n}, r_{j} \in \mathbb{R}^{2}, j=1,2$, $i=1, \ldots, n$,

$$
\left|B^{(1),(2)}(x, r, s)\right| \leq \Lambda_{2}|s|^{\varepsilon}+f_{j},
$$

where the functions $f_{j}$ are those from (2.3). On the functions $f_{j}$ in the right-hand sides of $(2.3)$ and $F_{j}$ in the structure conditions $(2.7 \mathrm{a})-(2.7 \mathrm{~b})$ the following conditions are imposed:

$$
\begin{gathered}
f_{1,2}(x, t) \in L^{\tau}(Q), \quad \tau>(p+n) / p, \\
F_{1,2}(x) \in L^{\theta}(Q), \quad \theta>(p+n) /(p-1) .
\end{gathered}
$$

3. The homogeneous system. The main idea is as follows. Instead of establishing estimates for each component $u^{j}$ of a solution separately we introduce some linear combinations of $u^{j}$ (in the general case these would be some functions $H^{j}(t, x, u)$ of $t, x$, and unknowns $\left.u^{j}\right)$, so that for each such linear combination (or function $H^{j}(t, x, u)$ ) the conventional maximum principle holds. Applying the classical maximum principle to the latter enables us to derive estimates for the components $u^{j}$ of the solution, in much the same way as for scalar equations. As an illustration consider the model system of two equations (2.3).

Let $H(u, v)$ be a twice continuously differentiable function to be determined later. Multiply the first equation by $H_{u}=\frac{\partial}{\partial u} H$ and add to the second one multiplied by $H_{v}=\frac{\partial}{\partial v} H$. This yields

$$
\begin{aligned}
& H_{u} u_{t}+H_{v} v_{t}=\frac{d}{d t} H \\
& =H_{u} \operatorname{div}\left(a_{1}|\nabla u|^{p-2} \nabla u\right)+H_{u} \operatorname{div}\left(b_{1}|\nabla v|^{p-2} \nabla v\right) \\
& \quad+H_{v} \operatorname{div}\left(a_{2}|\nabla u|^{p-2} \nabla u\right)+H_{v} \operatorname{div}\left(b_{2}|\nabla v|^{p-2} \nabla v\right)+f_{1} H_{u}+f_{2} H_{v} .
\end{aligned}
$$

To outline the basic idea we restrict ourselves to the case of a homogeneous system. For this case we shall obtain estimates of the solution $(u, v)$ by its values on the parabolic boundary $\partial Q$, i.e. the maximum principle. Suppose 
that $\sup _{Q} H$ is attained somewhere within $Q$. Multiply (3.1) by the test function $\varphi=(H-l)_{+}, l$ being the maximum value of $H(u, v)$ on the parabolic boundary $\partial Q$, and integrate with respect to $x$ over $\Omega$, and with respect to $t$ from 0 to $T$. This results in

$$
\begin{aligned}
& \frac{1}{2} \int_{A(T)} d x(H-l)^{2} \\
&=- \int_{0}^{T} d t \int_{A(t)} d x\left[a_{1}|\nabla u|^{p-2} \nabla u \cdot\left(H_{u u}^{2} \nabla u+H_{u v}^{2} \nabla v\right)\right. \\
&+b_{1}|\nabla v|^{p-2} \nabla v \cdot\left(H_{u u}^{2} \nabla u+H_{u v}^{2} \nabla v\right) \\
&+a_{2}|\nabla u|^{p-2} \nabla u \cdot\left(H_{u v}^{2} \nabla u+H_{v v}^{2} \nabla v\right) \\
&\left.+b_{2}|\nabla v|^{p-2} \nabla v \cdot\left(H_{u v}^{2} \nabla u+H_{v v}^{2} \nabla v\right)\right]
\end{aligned}
$$

where $A(t) \equiv\{H \geq l\}$. For convenience we shall write $\widetilde{H}$ for $H-l$. Let us now impose the following conditions on the derivatives of $\widetilde{H}(u, v)$ :

$$
\left\{\begin{array}{l}
a_{1} \widetilde{H}_{u u}^{2}+a_{2} \widetilde{H}_{u v}^{2}=\Lambda k^{p}, \\
a_{1} \widetilde{H}_{u v}^{2}+a_{2} \widetilde{H}_{v v}^{2}=\Lambda k^{p-1}, \\
b_{1} \widetilde{H}_{u u}^{2}+b_{2} \widetilde{H}_{u v}^{2}=\Lambda k, \\
b_{1} \widetilde{H}_{u v}^{2}+b_{2} \widetilde{H}_{v v}^{2}=\Lambda,
\end{array}\right.
$$

with $\Lambda=\Lambda(u, v) \geq 0, k=k(u, v)$; here and below, as usual it is understood that $k^{p}=|k|^{p}, k^{p-1}=|k|^{p-2} k$. First, multiply the first equation of (3.3) by $b_{1}$ and subtract the third multiplied by $a_{1}$ to get

$$
\left(a_{2} b_{1}-a_{1} b_{2}\right) \widetilde{H}_{u v}^{2}=\Lambda\left[b_{1} k^{p}-a_{1} k\right] .
$$

Multiplying the second equation of (3.3) by $b_{2}$ and subtracting the fourth multiplied by $a_{2}$ we obtain

$$
-\left(a_{2} b_{1}-a_{1} b_{2}\right) \widetilde{H}_{u v}^{2}=\Lambda\left[b_{2} k^{p-1}-a_{2}\right] .
$$

Hence we conclude that $k(u, v)$ is a solution to the equation

$$
k^{p}+\frac{b_{2}}{b_{1}} k^{p-1}-\frac{a_{1}}{b_{1}} k-\frac{a_{2}}{b_{1}}=0 .
$$

Now, subtracting from the first equation of (3.3) the second multiplied by $k$, and from the third one the fourth multiplied by $k$ we come to the system

$$
\left\{\begin{array}{l}
a_{1}\left(\widetilde{H}_{u u}^{2}-k \widetilde{H}_{u v}^{2}\right)+a_{2}\left(\widetilde{H}_{u v}^{2}-k \widetilde{H}_{v v}^{2}\right)=0 \\
b_{1}\left(\widetilde{H}_{u u}^{2}-k \widetilde{H}_{u v}^{2}\right)+b_{2}\left(\widetilde{H}_{u v}^{2}-k \widetilde{H}_{v v}^{2}\right)=0 .
\end{array}\right.
$$

From the ellipticity condition it follows that $a_{2} b_{1}-a_{1} b_{2} \not \equiv 0$ and thus

$$
\widetilde{H}_{u u}^{2}-k \widetilde{H}_{u v}^{2}=0, \quad \widetilde{H}_{u v}^{2}-k \widetilde{H}_{v v}^{2}=0 .
$$


Returning to our original notation we can rewrite this as

$$
\begin{aligned}
& (H-l)\left\{H_{u u}-k H_{u v}\right\}+\left\{\left(H_{u}\right)^{2}-k H_{u} H_{v}\right\}=0, \\
& (H-l)\left\{H_{u v}-k H_{v v}\right\}+\left\{H_{u} H_{v}-k\left(H_{v}\right)^{2}\right\}=0 .
\end{aligned}
$$

Since neither $k$ (as is seen from (3.4)) nor $H$ depend upon $l$, differentiating the first equation of (3.5) with respect to $l$ we obtain

$$
H_{u u}-k H_{u v}=0, \quad H_{u}-k H_{v}=0 .
$$

Differentiating the second equation of (3.6) with respect to $u$ yields

$$
H_{u u}-k H_{u v}-k_{u} H_{v}=0, \quad \text { so } \quad k_{u} H_{v} \equiv 0,
$$

and hence either $k_{u} \equiv 0$ or $H_{v} \equiv 0$. The choice $H_{v} \equiv 0$ would result in $H_{u} \equiv 0$ and $H=$ const, so we discard this possibility.

Differentiating the second equation of (3.5) with respect to $l$ yields

$$
H_{u v}-k H_{v v}=0, \quad H_{u}-k H_{v}=0 .
$$

Differentiating the second equation of (3.7) with respect to $v$ we have

$$
H_{u v}-k H_{v v}-k_{v} H_{v}=0, \quad \text { so } \quad k_{v} \equiv 0,
$$

and hence we conclude that

$$
k=\text { const }
$$

Solving the equation $H_{u}-k H_{v}=0$ we obtain $H=F(u k+v) ; F$ is any function. The simplest choice is $F(x) \equiv x$. Thus we are left with the following choice of $H$ :

$$
H=u k+v .
$$

In general (3.4) may have four roots; let us now determine conditions for at least two of them to be constant. From (3.4) we deduce that

$$
\left(k \frac{a_{1}}{b_{1}}+\frac{a_{2}}{b_{1}}\right) /\left(k+\frac{b_{2}}{b_{1}}\right)=k^{p-1} .
$$

Differentiating this with respect to $u$ and $v$, since $k=$ const, we arrive at

$$
\begin{aligned}
& k^{2}\left(\frac{a_{1}}{b_{1}}\right)_{u}^{\prime}+k\left[\left(\frac{b_{2}}{b_{1}}\right)^{2}\left(\frac{a_{1}}{b_{2}}\right)_{u}^{\prime}+\left(\frac{a_{2}}{b_{1}}\right)_{u}^{\prime}\right]+\left(\frac{b_{2}}{b_{1}}\right)^{2}\left(\frac{a_{2}}{b_{2}}\right)_{u}^{\prime}=0 \\
& k^{2}\left(\frac{a_{1}}{b_{1}}\right)_{v}^{\prime}+k\left[\left(\frac{b_{2}}{b_{1}}\right)^{2}\left(\frac{a_{1}}{b_{2}}\right)_{v}^{\prime}+\left(\frac{a_{2}}{b_{1}}\right)_{v}^{\prime}\right]+\left(\frac{b_{2}}{b_{1}}\right)^{2}\left(\frac{a_{2}}{b_{2}}\right)_{v}^{\prime}=0
\end{aligned}
$$


Set

$$
\begin{aligned}
A & =\left(\frac{a_{1}}{b_{1}}\right)_{u}^{\prime}=\left(\frac{a_{1}}{b_{1}}\right)_{v}^{\prime}, \\
B & =\left(\frac{b_{2}}{b_{1}}\right)^{2}\left(\frac{a_{1}}{b_{2}}\right)_{u}^{\prime}+\left(\frac{a_{2}}{b_{1}}\right)_{u}^{\prime}=\left(\frac{b_{2}}{b_{1}}\right)^{2}\left(\frac{a_{1}}{b_{2}}\right)_{v}^{\prime}+\left(\frac{a_{2}}{b_{1}}\right)_{v}^{\prime}, \\
C & =\left(\frac{b_{2}}{b_{1}}\right)^{2}\left(\frac{a_{2}}{b_{2}}\right)_{u}^{\prime}=\left(\frac{b_{2}}{b_{1}}\right)^{2}\left(\frac{a_{2}}{b_{2}}\right)_{v}^{\prime} \\
D & =\sqrt{B^{2}-4 A C}, \quad k_{1,2}=\frac{-B \pm \sqrt{B^{2}-4 A C}}{2 A} .
\end{aligned}
$$

One can see that though (3.4) in general may have four roots, since (3.9a) has at most two roots, the equation (3.4) with variable coefficients possesses at most two roots which are constants, and hence we get two choices of $H$ : $H_{1}=u k_{1}+v$ and $H_{2}=u k_{2}+v$. We come to the following conditions upon the coefficients $a_{1}, a_{2}, b_{1}, b_{2}$ :

$$
\begin{aligned}
& D(u, v)>0, \quad k_{1,2}=\text { const, } \\
& k_{1,2}^{p}+\frac{b_{2}(u, v)}{b_{1}(u, v)} k_{1,2}^{p-1}-\frac{a_{1}(u, v)}{b_{1}(u, v)} k_{1,2}-\frac{a_{2}(u, v)}{b_{1}(u, v)}=0, \\
& b_{1}(u, v) k_{1,2}+b_{2}(u, v)>0, \quad \forall(u, v) \in \mathbb{R} \times \mathbb{R} .
\end{aligned}
$$

Let $k_{1}$ and $k_{2}$ stand for the two different solutions of (3.4). We may assume that $k_{1}>k_{2}$. Now, with $H=H_{1}$ from (3.2) we have

$$
\begin{aligned}
& \int_{0}^{T} d t \int_{A(t)} d x H_{1 t}\left(H_{1}-l\right)=\frac{1}{2} \int_{A(T)} d x\left(H_{1}-l\right)^{2} \\
& \quad=-\Lambda \int_{0}^{T} d t \int_{A(t)} d x\left\langle\left|\nabla k_{1} u\right|^{p-2} \nabla k_{1} u+|\nabla v|^{p-2} \nabla v, \nabla\left(k_{1} u+v\right)\right\rangle,
\end{aligned}
$$

or

$$
\begin{aligned}
& \text { 10) } \frac{1}{2} \int_{A(T)} d x\left(H_{1}-l\right)^{2} \\
& +\Lambda \int_{0}^{T} d t \int_{A(t)} d x\left\langle\left|H_{1 u} \nabla u\right|^{p-2} \nabla u+\left|H_{1 v} \nabla v\right|^{p-2} \nabla v, H_{1 u} \nabla u+H_{1 v} \nabla v\right\rangle=0 .
\end{aligned}
$$

We shall make use of the following

Lemma 3.1. Let $p \geq 2$. Then for any $\vec{a}, \vec{b} \in \mathbb{R}^{n}$,

$$
\left\langle|\vec{a}|^{p-2} \vec{a}+|\vec{b}|^{p-2} \vec{b}, \vec{a}+\vec{b}\right\rangle \geq \frac{1}{2}\left[|\vec{a}|^{p-2}+|\vec{b}|^{p-2}\right]|\vec{a}+\vec{b}|^{2} \geq \frac{1}{2^{p-1}}|\vec{a}+\vec{b}|^{p},
$$

where the constant $1 / 2^{p-1}$ is sharp. 
For the proof of this lemma see e.g. [9, Lemma 4.4, p. 13].

Hence, it follows from (3.10) that

$$
\int_{0}^{T} d t \int_{A(t)} d x\left|\nabla\left(H_{1}\right)\right|^{p} \leq 0
$$

and by the embedding theorem we get

$$
C \int_{0}^{T} d t \int_{A(t)} d x\left|\left(H_{1}-l\right)\right|^{p} \leq \int_{0}^{T} d t \int_{A(t)} d x\left|\nabla\left(H_{1}-l\right)\right|^{p} \equiv \int_{0}^{T} d t \int_{A(t)} d x\left|\nabla H_{1}\right|^{p} \leq 0,
$$

where $C$ is constant. Thus we conclude that

$$
\sup _{Q} H_{1} \leq \sup _{\partial Q} H_{1} \text {. }
$$

Similarly, testing $(3.1)$ on the function $\varphi=\left(l-H_{1}\right)_{+}$, where $l$ now stands for the infimum of $H_{1}(u, v)$ on the parabolic boundary $\partial Q$, we obtain

$$
\inf _{Q} H_{1} \geq \inf _{\partial Q} H_{1}
$$

Analogously, with $H=H_{2}$ from (3.1) and (3.2) we arrive at the conclusion

$$
\sup _{Q} H_{2} \leq \sup _{\partial Q} H_{2}, \quad \inf _{Q} H_{2} \geq \inf _{\partial Q} H_{2} .
$$

Finally, we get the estimates

$$
\begin{aligned}
& \inf _{\partial Q} H_{1} \leq H_{1}=k_{1} u+v \leq \sup _{\partial Q} H_{1}, \\
& \inf _{\partial Q} H_{2} \leq H_{2}=k_{2} u+v \leq \sup _{\partial Q} H_{2},
\end{aligned}
$$

for a.e. $(x, t) \in Q$. This yields estimates for the unknowns themselves:

$$
\begin{aligned}
{\left[\inf _{\partial Q} H_{1}-\sup _{\partial Q} H_{2}\right] \cdot\left(k_{1}-k_{2}\right)^{-1} } & \leq u \leq\left[\sup _{\partial Q} H_{1}-\inf _{\partial Q} H_{2}\right] \cdot\left(k_{1}-k_{2}\right)^{-1}, \\
{\left[k_{1} \inf _{\partial Q} H_{2}-k_{2} \sup _{\partial Q} H_{1}\right] \cdot\left(k_{1}-k_{2}\right)^{-1} } & \leq v \\
& \leq\left[k_{1} \sup _{\partial Q} H_{2}-k_{2} \inf _{\partial Q} H_{1}\right] \cdot\left(k_{1}-k_{2}\right)^{-1}
\end{aligned}
$$

for a.e. $(x, t) \in Q$. Hence we immediately obtain estimates for $|u|$ and $|v|$ like

$$
|u| \leq \frac{1}{\left|k_{1}-k_{2}\right|} \max \left\{\left|\inf _{\partial Q} H_{1}-\sup _{\partial Q} H_{2}\right|,\left|\sup _{\partial Q} H_{1}-\inf _{\partial Q} H_{2}\right|\right\}
$$

or

$$
|u| \leq \frac{2}{\left|k_{1}-k_{2}\right|} \max \left\{\left|\sup _{\partial Q} H_{1}\right|,\left|\sup _{\partial Q} H_{2}\right|,\left|\inf _{\partial Q} H_{1}\right|,\left|\inf _{\partial Q} H_{2}\right|\right\},
$$


and

$$
|v| \leq \frac{1}{\left|k_{1}-k_{2}\right|} \max \left\{\left|k_{1} \inf _{\partial Q} H_{2}-k_{2} \sup _{\partial Q} H_{1}\right|,\left|k_{1} \sup _{\partial Q} H_{2}-k_{2} \inf _{\partial Q} H_{1}\right|\right\}
$$

or

$$
|v| \leq \frac{2}{\left|k_{1}-k_{2}\right|} \max \left\{\left|k_{2}\right|\left|\sup _{\partial Q} H_{1}\right|,\left|k_{1}\right| \operatorname{linf}_{\partial Q} H_{2}|,| k_{2}|| \inf _{\partial Q} H_{1}|,| k_{1}|| \sup _{\partial Q} H_{2} \mid\right\}
$$

for a.e. $(x, t) \in Q$. The latter can be rewritten in a concise form like

$$
|v|,|u| \leq C\left(\inf H_{1,2}, \sup H_{1,2}, k_{1,2}\right)
$$

for a.e. $(x, t) \in Q$.

4. Energy estimate of the solution. For the ongoing considerations we shall need an energy estimate for a solution of problem (2.1)-(2.2). Our goal in this section is to prove the following statement.

TheOREM 4.1. Let $(u, v)$ be a solution to problem (2.1)-(2.2). Then

$\underset{0<t<T}{\operatorname{ess} \sup _{\Omega}} \int_{\Omega}\left|u-\widetilde{u}_{0}\right|^{2}+\underset{0<t<T}{\operatorname{esssup}} \int_{\Omega}\left|v-\widetilde{v}_{0}\right|^{2}+\int_{0}^{T} \int_{\Omega}\left(\left|\nabla\left(u-\widetilde{u}_{0}\right)\right|^{p}+\left|\nabla\left(v-\widetilde{v}_{0}\right)\right|^{p}\right) \leq C$ and

$$
\int_{0}^{T} \int_{\Omega}\left(|\nabla u|^{p}+|\nabla v|^{p}\right) \leq C
$$

with some constant $C$ depending only on $\left\|f_{1,2}\right\|_{q^{\prime}, Q},\left\|\widetilde{u}_{0}\right\|_{\widetilde{W}(\partial Q)},\left\|\widetilde{v}_{0}\right\|_{\widetilde{W}(\partial Q)}$, $p, n, \lambda, \varepsilon$, mes $Q$, and independent of $u$ and $v$.

REMARK 2. In the formulation of the theorem and its proof, by $\widetilde{u}_{0}$ and $\widetilde{v}_{0}$ are meant any functions from $\widetilde{W}(Q)$ coinciding with $\widetilde{u}_{0}$ or $\widetilde{v}_{0}$ on the parabolic boundary. Therefore the final statement remains valid with the boundary norms.

Proof. A standard procedure is applied. Multiplying the first equation of (2.1) by $u-\widetilde{u}_{0}$ and the second one by $v-\widetilde{v}_{0}$, adding the results and integrating over $\Omega \times(0, t)$ we get

$$
\begin{aligned}
\int_{\Omega(t)} \frac{1}{2}\left(u-\widetilde{u}_{0}\right)^{2}+\int_{\Omega(t)} \frac{1}{2}\left(v-\widetilde{v}_{0}\right)^{2} & \\
& \quad+\int_{0}^{t} \int_{\Omega} \vec{A}^{(1)} \nabla\left(u-\widetilde{u}_{0}\right)+\int_{0}^{t} \int_{\Omega} \vec{A}^{(2)} \nabla\left(v-\widetilde{v}_{0}\right)
\end{aligned}
$$




$$
\begin{aligned}
& \leq \int_{0}^{t} \int_{\Omega}\left|B^{(1)}\right|\left|u-\widetilde{u}_{0}\right|+\int_{0}^{t} \int_{\Omega}\left|B^{(2)}\right|\left|v-\widetilde{v}_{0}\right| \\
& \quad+\int_{0}^{t}\left(\left|f_{1}\right|+\left|\widetilde{u}_{0 t}\right|\right)\left|u-\widetilde{u}_{0}\right|+\int_{0}^{t} \int_{\Omega}\left(\left|f_{2}\right|+\left|\widetilde{v}_{0 t}\right|\right)\left|v-\widetilde{v}_{0}\right|,
\end{aligned}
$$

where integration by parts with respect to the time variable was performed in the first two terms and the initial conditions were taken into account. By the ellipticity condition (2.4) and the growth (2.5) conditions on $A^{(1),(2)}$, the other two terms on the left can be bounded below as follows:

$$
\begin{aligned}
\int_{0}^{t} \int_{\Omega}\left(\vec{A}^{(1)}\right. & \left.\nabla\left(u-\widetilde{u}_{0}\right)+\vec{A}^{(2)} \nabla\left(v-\widetilde{v}_{0}\right)\right) \\
& =\int_{0}^{t}\left(\vec{A}_{\Omega}^{(1)} \nabla u+\vec{A}^{(2)} \nabla v-\vec{A}^{(1)} \nabla \widetilde{u}_{0}-\vec{A}^{(2)} \nabla \widetilde{v}_{0}\right) \\
& \geq \int_{0}^{t} \lambda\left(|\nabla u|^{p}+|\nabla v|^{p}\right)-\int_{0}^{t} \int_{\Omega} \lambda\left(|\nabla u|^{p-1}+|\nabla v|^{p-1}\right)\left(\left|\nabla u_{0}\right|+\left|\nabla v_{0}\right|\right) \\
& \geq \int_{0}^{t} \int_{\Omega} \frac{1}{2} \lambda\left(|\nabla u|^{p}+|\nabla v|^{p}\right)-\int_{0} \int_{\Omega} C(p, \lambda)\left(\left|\nabla u_{0}\right|^{p}+\left|\nabla v_{0}\right|^{p}\right) \\
& \geq \int_{0}^{t} \int_{\Omega} \frac{1}{2} \lambda\left(\left|\nabla\left(u-\widetilde{u}_{0}\right)\right|^{p}+\left|\nabla\left(v-\widetilde{v}_{0}\right)\right|^{p}\right)-\int_{0}^{t} \int_{\Omega} \widetilde{C}(p, \lambda)\left(\left|\nabla u_{0}\right|^{p}+\left|\nabla v_{0}\right|^{p}\right),
\end{aligned}
$$

with $\lambda=\lambda_{0}\left(1-\delta^{*} / 2\right)$. Here use has also been made of Young's inequality and the inequality

$$
|a+b|^{p} \leq C(p)\left(|a|^{p}+|b|^{p}\right), \quad \forall a, b \in \mathbb{R} .
$$

The first two terms on the right of (4.1), by virtue of the Young inequality, the Sobolev inequality and the growth condition (2.8), can be estimated as follows:

$$
\begin{aligned}
\int_{0}^{t} \int_{\Omega}\left|B^{(1)}\right|\left|u-\widetilde{u}_{0}\right|+\int_{0}^{t} \int_{\Omega} \mid & B^{(2)}|| v-\widetilde{v}_{0} \mid \\
& \leq C \int_{0}^{t} \int_{\Omega}(|\nabla u|+|\nabla v|)^{\varepsilon}\left(\left|u-\widetilde{u}_{0}\right|+\left|v-\widetilde{v}_{0}\right|\right) \\
& \leq \delta_{1} C_{1}(\varepsilon, p) \int_{0}^{t} \int_{\Omega}\left(\left|\nabla\left(u-\widetilde{u}_{0}\right)\right|+\left|\nabla\left(v-\widetilde{v}_{0}\right)\right|\right)^{p}
\end{aligned}
$$




$$
\begin{aligned}
+\delta_{2} C_{2}(p) \int_{0}^{t} \int_{\Omega}\left(\left|u-\widetilde{u}_{0}\right|+\right. & \left.\left|v-\widetilde{v}_{0}\right|\right)^{p}+C\left(C_{1,2}, \delta_{1,2}, \widetilde{u}_{0}, \widetilde{v}_{0}, \operatorname{mes} Q\right) \\
& \leq \delta_{3} \int_{0}^{t} \int_{\Omega}\left(\left|\nabla\left(u-\widetilde{u}_{0}\right)\right|+\left|\nabla\left(v-\widetilde{v}_{0}\right)\right|\right)^{p}+C_{3} .
\end{aligned}
$$

Here it has been taken into account that $\varepsilon / p+1 / p<1 / 2+1 / p \leq 1$. In much the same way, taking into account our hypotheses, we can estimate the last two integrals on the left-hand side of (4.1):

$$
\begin{aligned}
& \int_{0}^{t} \int_{\Omega}\left(\left|f_{1}\right|+\left|\widetilde{u}_{0 t}\right|\right)\left|u-\widetilde{u}_{0}\right|+\int_{0}^{t} \int_{\Omega}\left(\left|f_{2}\right|+\left|\widetilde{v}_{0 t}\right|\right)\left|v-\widetilde{v}_{0}\right| \\
& \quad \leq \int_{0}^{t}\left(\left|f_{1}\right|+\left|f_{2}\right|+\left|\widetilde{u}_{0 t}\right|+\left|\widetilde{v}_{0 t}\right|\right)\left(\left|u-\widetilde{u}_{0}\right|+\left|v-\widetilde{v}_{0}\right|\right) \\
& \leq\left\|\left|f_{1}\right|+\left|f_{2}\right|+\left|\widetilde{u}_{0 t}\right|+\left|\widetilde{v}_{0 t}\right|\right\|_{p^{\prime}, Q}\left(\int_{0}^{t} \int_{\Omega}\left(\left|u-\widetilde{u}_{0}\right|+\left|v-\widetilde{v}_{0}\right|\right)^{p}\right)^{1 / p} \\
& \leq \delta_{4} \int_{0}^{t} \int_{\Omega}\left(\left|\nabla\left(u-\widetilde{u}_{0}\right)\right|+\left|\nabla\left(v-\widetilde{v}_{0}\right)\right|\right)^{p}+C_{4}\left(\operatorname{mes} Q, f_{1,2}, \delta_{4}, \widetilde{u}_{0}, \widetilde{v}_{0}\right) .
\end{aligned}
$$

Collecting the above estimates, from (4.1) we get

$$
\begin{aligned}
& \int_{\Omega(t)} \frac{1}{2}\left[\left(u-\widetilde{u}_{0}\right)^{2}+\left(v-\widetilde{v}_{0}\right)^{2}\right]+\int_{0}^{t} \int_{\Omega} \frac{1}{2} \lambda\left(\left|\nabla\left(u-\widetilde{u}_{0}\right)\right|^{p}+\left|\nabla\left(v-\widetilde{v}_{0}\right)\right|^{p}\right) \\
& \leq \delta_{5} \int_{0}^{t} \int_{\Omega}\left(\left|\nabla\left(u-\widetilde{u}_{0}\right)\right|^{p}+\left|\nabla\left(v-\widetilde{v}_{0}\right)\right|^{p}\right)+C_{4}\left(\operatorname{mes} Q, f_{1,2}, \delta_{5}, \widetilde{u}_{0}, \widetilde{v}_{0}\right) .
\end{aligned}
$$

Choosing $\delta_{5}=\frac{1}{4} \lambda$ yields

$$
\begin{array}{r}
\int_{\Omega(t)} \frac{1}{2}\left[\left(u-\widetilde{u}_{0}\right)^{2}+\left(v-\widetilde{v}_{0}\right)^{2}\right]+\int_{0}^{t} \int_{\Omega} \frac{1}{4} \lambda\left(\left|\nabla\left(u-\widetilde{u}_{0}\right)\right|^{p}+\left|\nabla\left(v-\widetilde{v}_{0}\right)\right|^{p}\right) \\
\leq C_{4}\left(f_{1,2}, \delta_{5}, \widetilde{u}_{0}, \widetilde{v}_{0}\right) .
\end{array}
$$

Now we take the supremum over $t$ on the right-hand side of (4.4) to obtain

$$
\begin{aligned}
\underset{0<t<T}{\operatorname{ess} \sup _{\Omega}} \int_{\Omega}\left|u-\widetilde{u}_{0}\right|^{2}+\underset{0<t<T}{\operatorname{ess} \sup } \int_{\Omega} & \left|v-\widetilde{v}_{0}\right|^{2} \\
& +\int_{0}^{T} \int_{\Omega}\left(\left|\nabla\left(u-\widetilde{u}_{0}\right)\right|^{p}+\left|\nabla\left(v-\widetilde{v}_{0}\right)\right|^{p}\right) \leq C_{5}
\end{aligned}
$$


with a constant $C_{5}$ depending on $n, p, \varepsilon, \lambda,\left\|f_{1,2}\right\|_{p^{\prime}, Q}$, mes $Q$ and, by Remark 2 , the boundary norms $\left\|\widetilde{u}_{0}\right\|_{\widetilde{W}(\partial Q)}$ and $\left\|\widetilde{v}_{0}\right\|_{\widetilde{W}(\partial Q)}$ of the functions in the boundary conditions only. Hence the second statement of the theorem is self-evident.

5. The main result. Let us now turn our attention to the general nonhomogeneous system whose coefficients satisfy the structure hypotheses $(2.7 \mathrm{a})-(2.7 \mathrm{~b})$. Our main result is

Theorem 5.1. Let $(u, v)$ be a solution to problem (2.1)-(2.2). If hypotheses $(2.7 \mathrm{a})-(2.7 \mathrm{~b})$ along with assumptions (2.6a)-(2.6d) are fulfilled, i.e. there exist two different numbers $k_{1}, k_{2}$ satisfying equation (3.4) with $\Lambda_{1}>0$ and $\Lambda_{2}>0$ so that (2.7a)-(2.7b) hold with precisely those $k$, then for the two linearly independent functions $\mathrm{H}_{1}$ and $\mathrm{H}_{2}$ defined by (3.8) we have the estimates

$$
\left\|H_{1}\right\|_{L_{\infty}(Q)} \leq C_{1}, \quad\left\|H_{2}\right\|_{L_{\infty}(Q)} \leq C_{2} .
$$

Hence the same estimates hold for the components of the solution:

$$
\|u\|_{L_{\infty}(Q)} \leq C_{1}, \quad\|v\|_{L_{\infty}(Q)} \leq C_{2},
$$

where the constants $C_{1,2}$ depend only on $p, n, f_{1,2}, F_{1,2}, a_{1,2}(s), b_{1,2}(s)$, $\left|g_{1,2}\right|_{\infty,(S)},\left|u_{0}, v_{0}\right|_{\infty,(\Omega)},\left\|\widetilde{u}_{0}\right\|_{\widetilde{W}(\partial Q)},\left\|\widetilde{v}_{0}\right\|_{\widetilde{W}(\partial Q)}$, the domain $Q$, and constants in embedding theorems, and are independent of $u$ and $v$.

To prove the theorem we need Stampacchia's well known lemma:

LEMma 5.2. Let $\psi(y)$ be a nonnegative nondecreasing function defined on $\left[l_{0}, \infty\right)$ which satisfies

$$
\psi(m) \leq \frac{C}{(m-l)^{\vartheta}}\{\psi(l)\}^{\delta} \quad \text { for } \quad m>l \geq l_{0},
$$

with $\vartheta>0$ and $\delta>1$. Then

$$
\psi\left(l_{0}+d\right)=0,
$$

where $d=C^{1 / \vartheta}\left\{\psi\left(k_{0}\right)\right\}^{(\delta-1) / \vartheta} 2^{\delta /(\delta-1)}$.

For the proof see $[7$, Lemma 4.1, p. 8]. We also make use of the following lemma (see [9, Prop. 3.1, p. 7]):

Lemma 5.3. If $u \in L^{\infty}\left(0, T ; L^{2}(\Omega)\right) \cap L^{p}\left(0, T ; W_{0}^{1, p}(\Omega)\right)$ then

$$
\int_{0}^{T} \int_{\Omega} u^{q} \leq C\left(\int_{0}^{T} \int_{\Omega}|\nabla u|^{p}\right)\left(\underset{0<t<T}{\operatorname{ess} \sup _{\Omega}}|u|^{2}\right)^{p / n}
$$

with $q=p(n+2) / n$ and the constant $C$ depending only on $p$ and $n$.

Proof of Theorem 5.1. Let $k_{1}$ be a solution to (3.4). Multiply the first equation of (2.1) by $k_{1}$, add to the second, and choose $w \equiv \operatorname{sign} H_{1}\left(\left|H_{1}\right|-l\right)_{+}$ 
as a test function with $l \geq l_{0}=\max \left[\left\|k_{1} g_{1}+g_{2}\right\|_{L^{\infty}(S)},\left\|k_{1} u_{0}+v_{0}\right\|_{L^{\infty}(\Omega)}\right]$. After integrating in $t$ from 0 to $t, t \leq T$, and in $x$ over $\Omega$, this results in

$$
\begin{aligned}
& \frac{1}{2} \int_{\Omega(t)} w^{2}+\int_{0}^{t} \int_{\Omega}\left\langle k_{1} \vec{A}^{(1)}\left(x, u, v, u_{x}, v_{x}\right)+\vec{A}^{(2)}\left(x, u, v, u_{x}, v_{x}\right), \nabla w\right\rangle \\
& \quad=\int_{0}^{t} \int_{\Omega}\left(k_{1} B^{(1)}\left(x, u, v, u_{x}, v_{x}\right)+B^{(2)}\left(x, u, v, u_{x}, v_{x}\right)\right) w+\int_{0}^{t} \int_{\Omega}\left(f_{1} k_{1}+f_{2}\right) w .
\end{aligned}
$$

From this identity, by the hypotheses on the leading part (2.7a)-(2.7b), and the growth assumptions (2.8) on the right-hand sides, we have

$$
\begin{aligned}
\frac{1}{2} \int_{\Omega(t)} w^{2}+\Lambda_{1} \int_{0}^{t} \int_{\Omega}\left\langle\left|k_{1}\right|^{p-2} k_{1}|\nabla u|^{p-2} \nabla u-|\nabla v|^{p-2} \nabla v, \nabla w\right\rangle \\
\leq \varepsilon_{1} k_{1}\left(1-\frac{\left|k_{2}\right|}{\left|k_{1}\right|}\right) \int_{0}^{t} \int_{\Omega}\left(|\nabla u|^{p-2}+|\nabla v|^{p-2}\right)|\nabla w|^{2} \\
\quad+\int_{0}^{t} \int_{\Omega}\left|F_{1}\right||\nabla w|+C \int_{0}^{t} \int_{\Omega}(|\nabla u|+|\nabla v|)^{\varepsilon} w+\int_{0}^{t} \int_{\Omega} f w,
\end{aligned}
$$

where $f=f_{1} k_{1}+f_{2}, C=C\left(\lambda, k_{1}, p\right)$ is a constant. Since $t \in(0, T]$ is arbitrary, taking the supremum over $t$ and applying Lemma 3.1 to the second term on the left, we get

$$
\begin{aligned}
& \sup _{0<t<T} \int_{\Omega} w^{2}+\frac{\Lambda_{1}}{2} \int_{0}^{T} \int_{\Omega}\left(|\nabla u|^{p-2}+|\nabla v|^{p-2}\right)|\nabla w|^{2} \\
& \leq \varepsilon_{1} k_{1}\left(1-\frac{\left|k_{2}\right|}{\left|k_{1}\right|}\right) \int_{0}^{T} \int_{\Omega}\left(|\nabla u|^{p-2}+|\nabla v|^{p-2}\right)|\nabla w|^{2} \\
&+\int_{0}^{T} \int_{\Omega}\left|F_{1}\right||\nabla w|+C \int_{0}^{T} \int_{\Omega}(|\nabla u|+|\nabla v|)^{\varepsilon} w+\int_{0}^{T} \int_{\Omega} f w ;
\end{aligned}
$$

hence, taking into account the conditions on $\varepsilon_{1,2}$ in hypotheses $(2.7 \mathrm{a})-(2.7 \mathrm{~b})$, we infer that

$$
\sup _{0<t<T} \int_{\Omega} w^{2}+\int_{0}^{T} \int_{\Omega}|\nabla w|^{p} \leq C_{1} \int_{0}^{T} \int_{\Omega}\left|F_{1} \| \nabla w\right|+C_{2} \int_{0}^{T} \int_{\Omega}(|\nabla u|+|\nabla v|)^{\varepsilon}+\int_{0}^{T} \int_{\Omega} f w,
$$

where $C_{1}$ and $C_{1}$ are some constants depending on $\varepsilon_{1}, k_{1,2}, \Lambda_{1}, p$ and $n$. Applying the generalized Hölder inequality to the three terms on the right yields 


$$
\begin{aligned}
\sup _{0<t<T} \int_{\Omega} w^{2} & +\int_{0}^{T} \int_{\Omega}|\nabla w|^{p} \\
\leq & C_{1}\|\nabla w\|_{p, Q}\left\|F_{1}\right\|_{\theta, Q}\left(\int_{0}^{T} \int_{\Omega} \chi_{A(l)}\right)^{1-1 / p-1 / \theta} \\
& +C_{2}\|w\|_{q, Q}\|f\|_{r, Q}\left(\int_{0}^{T} \int_{\Omega} \chi_{A(l)}\right)^{1-1 / q-1 / r} \\
& +C_{3}\|w\|_{q, Q}\left\||\nabla u|^{\varepsilon}+|\nabla v|^{\varepsilon}\right\|_{p / \varepsilon, Q}\left(\int_{0}^{T} \int_{\Omega} \chi_{A(l)}\right)^{1-1 / q-\varepsilon / p}
\end{aligned}
$$

where $\chi_{A(l)}$ is the characteristic function of the set $A(l)$, and $r$ has been selected such that $\tau>r>(p+n) / p$. From Lemma 5.3 it follows that

$$
\|w\|_{q, Q} \leq\left(\sup _{0<t<T} \int_{\Omega} w^{2}+\int_{0}^{T} \int_{\Omega}|\nabla w|^{p}\right)^{(p+n) / q n} .
$$

From (5.1) and this inequality we obtain

$$
\begin{aligned}
\sup _{0<t<T} \int_{\Omega} w^{2}+\int_{0}^{T} \int_{\Omega}|\nabla w|^{p} \\
\leq C_{1}\left\|F_{1}\right\|_{\theta, Q}\left(\sup _{0<t<T} \int_{\Omega} w^{2}+\int_{0}^{T} \int_{\Omega}|\nabla w|^{p}\right)^{1 / p}\{\psi(l)\}^{1-1 / p-1 / \theta} \\
\quad+C_{2}\left(\sup _{0<t<T} \int_{\Omega} w^{2}+\int_{0}^{T} \int_{\Omega}|\nabla w|^{p}\right)^{(n+p) / n q}\|f\|_{r, Q}\{\psi(l)\}^{1-1 / q-1 / r} \\
\quad+C_{3}\left(\sup _{0<t<T} \int_{\Omega} w^{2}+\int_{0}^{T}|\nabla w|_{\Omega}^{p}\right)^{(n+p) / n q} \\
\quad \times\left\||\nabla u|^{\varepsilon}+|\nabla v|^{\varepsilon}\right\|_{p / \varepsilon, Q}\{\psi(l)\}^{1-1 / q-\varepsilon / p}
\end{aligned}
$$

where we have set for brevity

$$
\psi(l)=\int_{0}^{T} \operatorname{mes} A\{w \geq l\}(l, t) d t .
$$

Applying the Young inequality to the right-hand side of (5.3) gives

$$
\begin{aligned}
\sup _{0<t<T} \int_{\Omega} w^{2}+\int_{0}^{T} \int_{\Omega}|\nabla w|^{p} \leq C_{1}\{\psi(l)\}^{(1-1 / p-1 / \theta)\left(\frac{p}{p-1}\right)} \\
+C_{2}\|f\|_{(p+n) / p, Q}\{\psi(l)\}^{(1-1 / q-1 / r)\left(\frac{n q}{n+p}\right) \#} \\
+C_{3}\left\||\nabla u|^{\varepsilon}+|\nabla v|^{\varepsilon}\right\|_{p / \varepsilon, Q}\{\psi(l)\}^{(1-1 / q-\varepsilon / p)\left(\frac{n q}{n+p}\right)^{\#}} .
\end{aligned}
$$


By the second statement of Theorem 4.1 we can estimate

$$
\left\||\nabla u|^{\varepsilon}+|\nabla v|^{\varepsilon}\right\|_{p / \varepsilon, Q} \leq \widetilde{C}\left(\int_{0}^{T} \int_{\Omega}|\nabla u|^{p}+\int_{0}^{T} \int_{\Omega}|\nabla v|^{p}\right)^{\varepsilon / p} \leq C\left(u_{0}, v_{0}, g_{1,2}\right),
$$

which applied to (5.4) results in

$$
\begin{aligned}
\sup _{0<t<T} \int_{\Omega} w^{2}+\int_{0}^{T} \int_{\Omega}|\nabla w|^{p} \leq C_{1}\{\psi(l)\}^{(1-1 / p-1 / \theta)\left(\frac{p}{p-1}\right)} \\
+C_{2}\{\psi(l)\}^{(1-1 / q-1 / r)\left(\frac{n q}{n+p}\right)^{\#}}+C_{3}\{\psi(l)\}^{(1-1 / q-\varepsilon / p)\left(\frac{n q}{n+p}\right)^{\#}}
\end{aligned}
$$

with $C_{1}=C\left(F_{1,2}, p, \theta, \lambda_{1}\right), C_{2}=C\left(p, n, \lambda_{1}, f_{1,2}\right)$ and $C_{3}=C\left(p, n, \lambda_{1}, u_{0}, v_{0}\right.$, $\left.g_{1,2}\right)$. Resorting again to (5.2) we obtain

$$
\begin{aligned}
& \left(\|w\|_{q, Q}\right)^{n q /(n+p)} \leq C_{1}\{\psi(l)\}^{(1-1 / p-1 / \theta)\left(\frac{p}{p-1}\right)} \\
& \quad+C_{2}\{\psi(l)\}^{(1-1 / q-1 / r)\left(\frac{n q}{n+p}\right)^{\#}}+C_{3}\{\psi(l)\}^{(1-1 / q-\varepsilon / p)\left(\frac{n q}{n+p}\right)^{\#}} .
\end{aligned}
$$

Let us estimate

$$
(m-l)\{\psi(m)\}^{1 / q}=(m-l)\left(\int_{0}^{T} \int_{\Omega} \chi_{A(m)}\right)^{1 / q}<\left(\int_{0}^{T} \int_{\Omega} w^{q} \chi_{A(m)}\right)^{1 / q}<\|w\|_{q, Q},
$$

where $m>l \geq l_{0}$. Substituting this into (5.5) we come to

$$
\begin{aligned}
(m-l)^{q} \psi(m) \leq & C_{1}\{\psi(l)\}^{(1-1 / q-1 / r)\left(\frac{n q}{n+p}\right) \#\left(\frac{n+p}{n}\right)} \\
& +C_{2}\{\psi(l)\}^{(1-1 / p-1 / \theta)\left(\frac{p(n+p)}{n(p-1)}\right)} \\
& +C_{3}\{\psi(l)\}^{(1-1 / q-\varepsilon / p)\left(\frac{n q}{n+p}\right) \#\left(\frac{n+p}{n}\right)}
\end{aligned}
$$

or, succinctly,

$$
\psi(m) \leq \frac{C_{1}}{(m-l)^{q}}\{\psi(l)\}^{\delta_{1}}+\frac{C_{2}}{(m-l)^{q}}\{\psi(l)\}^{\delta_{2}}+\frac{C_{3}}{(m-l)^{q}}\{\psi(l)\}^{\delta_{3}}
$$

with

$$
\begin{aligned}
& \delta_{1}=\left(1-\frac{n}{p(n+2)}-\frac{1}{r}\right) /\left(\frac{n}{n+p}-\frac{n}{p(n+2)}\right), \\
& \delta_{2}=\left(\frac{p-1}{p}-\frac{1}{\theta}\right) \frac{p(n+p)}{n(p-1)}, \\
& \delta_{3}=\left(1-\frac{n}{p(n+2)}-\frac{\varepsilon}{p}\right) /\left(\frac{n}{n+p}-\frac{n}{p(n+2)}\right) .
\end{aligned}
$$

Since we assume that

$$
f_{1,2} \in L^{\tau}(Q), \quad \tau>p /(p-1),
$$

it is not difficult to check that

$$
1-\frac{n}{p(n+2)}-\frac{1}{r}>\frac{n}{n+p}-\frac{n}{p(n+2)}, \quad \text { and hence } \quad \delta_{1}>1 .
$$


In much the same way, from the hypothesis

$$
F_{1,2} \in L^{\theta}(Q), \quad \theta>(p+n) /(p-1),
$$

it follows that

$$
\frac{p-1}{p}-\frac{1}{\theta}>\frac{n(p-1)}{p(n+p)}, \quad \text { thus } \quad \delta_{2}>1 ;
$$

and, since we assume $\varepsilon<p^{2} /(n+p)$,

$$
1-\frac{n}{p(n+2)}-\frac{\varepsilon}{p}>\frac{n}{n+p}-\frac{n}{p(n+2)}, \quad \text { and hence } \quad \delta_{3}>1 .
$$

Without loss of generality we may assume that $\psi(l)<1$. In fact, from the first statement of Theorem 4.1 and (5.2) it follows that

$$
\begin{aligned}
& \left(l-l_{0}\right)\{\psi(l)\}^{1 / q}=\left(l-l_{0}\right)\left(\int_{0}^{T} \int_{\Omega} \chi_{A(l)}\right)^{1 / q}<\left(\int_{0}^{T} \int_{\Omega}\left(H_{1}-l_{0}\right)^{q} \chi_{A(l)}\right)^{1 / q} \\
& <\left\|H_{1}-l_{0}\right\|_{q, Q} \leq\left(\sup _{0<t<T} \int_{\Omega}\left(H_{1}-l_{0}\right)^{2}+\int_{0}^{T} \int_{\Omega}\left|\nabla\left(H_{1}-l_{0}\right)\right|^{p}\right)^{\frac{p+n}{q n}} \leq \widetilde{C},
\end{aligned}
$$

with $l \geq l_{0}$, and hence

$$
\psi(l) \leq \widetilde{C}^{q} /\left(l-l_{0}\right)^{q} ;
$$

and it is easy to see that $\psi(l)<1$ whenever $l$ is chosen such that $l>\widetilde{C}+l_{0}$. Since $\psi(l)$ is a nonincreasing function, $\psi(l)<1$ remains true for all numbers $l>\widetilde{C}+l_{0}$.

Due to this fact, inequality (5.6) yields

$$
\psi(m) \leq \frac{C}{(m-l)^{q}}\{\psi(l)\}^{\delta}
$$

with $\delta=\min \left[\delta_{1}, \delta_{2}, \delta_{3}\right]$ and $C=\max \left[C_{1}, C_{2}, C_{3}\right]$. By Lemma 5.2 from (5.7) we conclude that

$$
\psi\left(l_{0}+d\right)=0
$$

for some $d$ sufficiently large, but finite, depending only on $p, n, \eta_{1}, f_{1,2}, F_{1,2}$, $u_{0}, v_{0}, g_{1,2}, a_{i}, b_{i}$, the domain $Q$, and constants in embedding theorems, and independent of $u$ and $v$. Analogously we proceed for $H_{2}=k_{2} u+v$, where $k_{2}$ stands for another solution to (3.4).

It is not difficult to see from the previous considerations that the same estimates hold for the components $(u, v)$ of the solution. In fact,

$$
\begin{aligned}
\|u\|_{\infty} & =\left\|k_{1} u-k_{2} u\right\|_{\infty} /\left|k_{1}-k_{2}\right|=\left\|\left(k_{1} u+v\right)-\left(k_{2} u+v\right)\right\|_{\infty} /\left|k_{1}-k_{2}\right| \\
& =\left\|H_{1}-H_{2}\right\|_{\infty} /\left|k_{1}-k_{2}\right| \leq\left(C_{1}+C_{2}\right) /\left|k_{1}-k_{2}\right|, \\
\|v\|_{\infty} & =\left\|v+k_{1} u-k_{1} u\right\|_{\infty} \leq\left\|k_{1} u\right\|_{\infty}+\left\|k_{1} u+v\right\|_{\infty}=k_{1}\|u\|_{\infty}+\left\|H_{1}\right\|_{\infty},
\end{aligned}
$$

and hence the statement follows. 


\section{References}

[1] H. Amann, Dynamic theory of quasilinear parabolic equations. I. Abstract evolution equations, Nonlinear Anal. 12 (1988), 895-919.

[2] -, Dynamic theory of quasilinear parabolic systems. III. Global existence, Math. Z. 202 (1989), 219-250; Erratum, ibid. 205 (1990), 331.

[3] —, Dynamic theory of quasilinear parabolic equations. II. Reaction-diffusion systems, Differential Integral Equations 3 (1990), 13-75.

[4] A. V. Bitsadze, On elliptic systems of differential equations with partial derivatives of second order, Dokl. Akad. Nauk SSSR 112 (1957), 983-986 (in Russian).

[5] -, Boundary Value Problems for Second Order Elliptic Equations, Nauka, Moscow, 1966 (in Russian).

[6] —, Some Classes of Partial Differential Equations, Nauka, Moscow, 1981 (in Russian).

[7] Y. Z. Chen and L. C. Wu, Second Order Elliptic Equations and Elliptic Systems, Amer. Math. Soc., Providence, RI, 1998.

[8] E. De Giorgi, Un esempio di estremali discontinue per un problema variazionale di tipo ellittico, Boll. Un. Mat. Ital. 1 (1968), 135-137.

[9] E. DiBenedetto, Degenerate Parabolic Equations, Springer, New York, 1993.

[10] L. Dung, Hölder regularity for certain strongly coupled parabolic systems, J. Differential Equations 151 (1999), 313-344.

[11] M. Giaquinta, Introduction to the Theory of Nonlinear Elliptic Systems, Birkhäuser, Berlin, 1991.

[12] M. Giaquinta and J. Souček, Caccioppoli's inequality and Legendre-Hadamard condition, Math. Ann. 270 (1985), 105-107.

[13] K. H. W. Küfner, Global existence for a certain strongly coupled quasilinear parabolic system in population dynamics, Analysis 15 (1995), 343-357.

[14] O. A. Ladyzhenskaya, N. A. Solonnikov, and N. N. Ural'tseva, Linear and Quasilinear Equations of Parabolic Type, Amer. Math. Soc., Providence, RI, 1968.

[15] D. Portnyagin, A generalization of the maximum principle to nonlinear parabolic systems, Ann. Polon. Math. 81 (2003), 217-236.

[16] M. A. Pozio and A. Tesei, Global existence of solutions for a strongly coupled quasilinear parabolic system, Nonlinear Anal. 12 (1990), 657-689.

[17] M. Wiegner, Global solutions to a class of strongly coupled parabolic systems, Math. Ann. 292 (1992), 711-727.

Institute for Condensed Matter Physics

of the National Academy of Sciences of Ukraine

1 Svientsitskii St.

79011 Lviv, Ukraine

E-mail: port@icmp.lviv.ua 